\title{
Gesture Controller for Robotic Vehicle
}

\section{Piyush Valtaprasad Kshatriya*}

Department of Robotics, Maharaja Sayajirao University of Baroda, Vadodara, Gujarat, India

\begin{abstract}
In this assignment we have used hand development to drive the robot. Hence we have used accelerometer which tackles enlivening. A movement controlled robot is controlled by using hand rather than some other technique like gets or joystick. Here one simply needs to move hand to control the robot. A transmitting device is used in your grip which contains RF Transmitter and accelerometer. This will transmit bring to robot with the objective that it can do the required task like pushing ahead, modify, turning left, turning right and stop.
\end{abstract}

\section{Introduction}

Robots are assuming a critical part in mechanization over every one of the segments like development, military, restorative, fabricating, and so on. In the wake of making some essential robots like line supporter robot, PC controlled robot, and so forth. We have built up this accelerometer based signal controlled robot by utilizing Arduino Uno. In this task we have utilized hand movement to drive the robot. For this reason we have utilized accelerometer which takes a shot at quickening. A motion controlled robot is controlled by utilizing hand instead of some other strategy like catches or joystick. Here one just needs to move hand to control the robot. A transmitting gadget is utilized in your grasp which contains RF Transmitter and accelerometer. This will transmit summon to robot with the goal that it can do the required assignment like pushing ahead, invert, turning left, turning right and stop. Every one of these assignments will be performed by utilizing hand motion. Here the most critical part is accelerometer. Accelerometer is a 3 hub increasing speed estimation gadget with $+-3 \mathrm{~g}$ territory. This gadget is made by utilizing polysilicon surface sensor and flag moulding circuit to gauge quickening. The yield of this gadget is Analog in nature and relative to the increasing speed. This gadget estimates the static increasing speed of gravity when we tilt it. Also, gives an outcome in type of movement or vibration.

\section{Block Diagram}

The square graph of the remote signal controlled robot is appeared as takes after. The circuit outline of the transmitter area of the remote motion controlled robot is appeared in Figure 1.

In this venture, a hand signal controlled robot is created utilizing 3-pivot Accelerometer sensor and Arduino. Rather than remote with catches or a joystick, the signals of the hand are utilized to control the movement of the robot. The task depends on remote correspondence and the undertaking is partitioned into transmitter and collector area $[1,2]$.

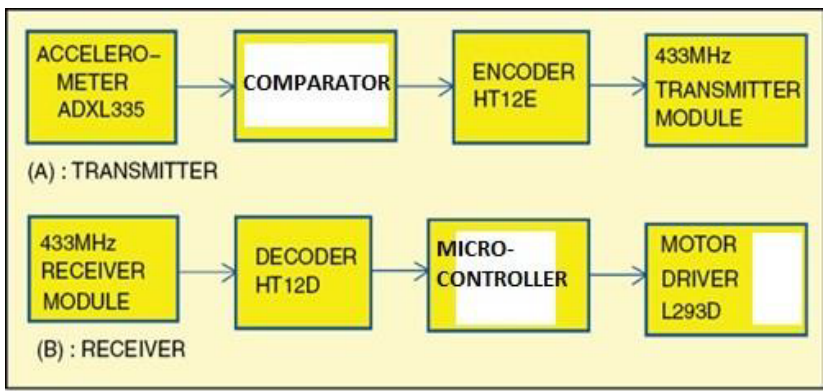

Figure 1: Block diagram of signal controlled and remote controlled robot.

\section{List of components}

\section{Axis accelerometer}

- It is an electromechanical gadget that estimates the increasing speed or g-constrain. Accelerometer and Gyroscope are the two tangible gadgets that are utilized to decide the position and introduction of a protest (Figure 2).

- Accelerometer estimates the static speeding up or nonGravitational quickening of a question like in tilting a protest. It can likewise gauge the dynamic speeding up when a question picks up a speed from halt because of development, vibrations or stun. All the most recent advanced cells are furnished with accelerometers to change the introduction of the show in view of the tilt of the telephone (Figure 3).

- RF Transmitter and Receiver Modules [3,4].

- The correspondence amongst transmitter and recipient is utilizing RF modules.

- A $434 \mathrm{MHz}$ transmitter and collector match are utilized in this venture.

\begin{tabular}{|c|c|}
\hline Component & Part No / Value / Keyword \\
\hline comparator & \\
\hline IC1 & 3-Axis Accelerometer \\
\hline IC2 & HT-12E \\
\hline IC3 & RF Transmitter \\
\hline R1 & $1.1 \mathrm{M} \Omega$ \\
\hline
\end{tabular}

Figure 2: Components for Transmitter Section.

${ }^{*}$ Corresponding author: Piyush Valtaprasad Kshatriya, Department of Robotics, Maharaja Sayajirao University of Baroda, Vadodara, Gujarat, India, Tel: +918200079638; E-mail: kshatriyapiyush@yahoo.com

Received September 02, 2018; Accepted September 28, 2018; Published October 05, 2018

Citation: Kshatriya PV (2018) Gesture Controller for Robotic Vehicle. Adv Robot Autom 7: 188. doi: 10.4172/2168-9695.1000188

Copyright: (c) 2018 Kshatriya PV. This is an open-access article distributed under the terms of the Creative Commons Attribution License, which permits unrestricted use, distribution, and reproduction in any medium, provided the original author and source are credited. 


\begin{tabular}{|c|c|}
\hline Component & Keyword \\
\hline IC1 & HT-12D \\
\hline IC2 & L293D \\
\hline IC3 & RF Receiver \\
\hline M1 and M2 & wheel motors \\
\hline R1 & $1 \mathrm{~K} \Omega$ \\
\hline R2 & $51 \mathrm{~K} \Omega$ \\
\hline LED1 & Red LED \\
\hline Robot Chassis & Arduino uno \\
\hline
\end{tabular}

Figure 3: Components for Receiver Section.

\section{HT-12E}

It is an encoder IC that changes over the 4-bit parallel information into serial information with a specific end goal to transmit over RF connect.

\section{HT-12D}

- It is a decoder IC that changes over the serial information gotten by the RF Receiver into 4-bit parallel information. This parallel information can be utilized to drive the engines.

- Working of Hand Gesture Controlled Robot

- In this venture, a versatile robot that is controlled by the motions made by the hand is outlined. The working of the robot is clarified here.

- As said before, the motion controlled robot is a remote worked robot and has two sections: Transmitter and Receiver. At the point when the robot is fuelled on, the transmitter part, which comprises of Comparator, Accelerometer, Encoder and RF Transmitter, will ceaselessly screen the accelerometer sensor.

- This information is caught by the Comparator, which transmits proper information to the Encoder, in light of the introduction of the Accelerometer.

- The parallel information gotten by the encoder is changed over into serial information and this serial information is transmitted by the RF Transmitter.

- At the recipient segment, the RF Receiver gets the serial information and transmits to Decoder IC. The Decoder will change over the serial information to parallel information and this parallel information is given to the Arduino as information and yield is given to the engine driver IC. In light of the information, the development robot is characterized [5].

\section{Result}

1. Transmission through RF (Radio recurrence) is superior to IR (infrared) due to numerous reasons.

2. Firstly, motions through RF can go through bigger separations making it appropriate for long range applications.

3. Also, while IR for the most part works in observable pathway mode, RF signs can travel notwithstanding when there is a hindrance between transmitter and recipient.

4. Next, RF transmission is more solid and dependable than IR transmission.

\section{References}

1. Fegade $T$, Kurle $Y$, Nikale $S$, Kalpund $P$ (2016) Wireless gesture controlled Semi-Humanoid Robot. International Conference on Recent Advances and Innovations in Engineering (ICRAIE).

2. Vivek Krishna R, Sathish BS, Ganesan P, Babu JR (2015) Design of voice and gesture controlled Quadcopter. International Conference on Innovations in Information, Embedded and Communication Systems (ICIIECS).

3. Xu A, Dudek G, Sattar J (2008) A natural gesture interface for operating robotic systems. IEEE International Conference on Robotics and Automation.

4. https://www.instructables.com/id/Gesture-controlled-robot-using-Arduino.

5. https://www.hackster.io/mayooghgirish/hand-gesture-controlled-robot-4d7587. 